\title{
Carnets
}

Revue électronique d'études françaises de l'APEF

Deuxième série - 3 | 2015

Insulaire

\section{L'insularité : du mythe à la réalité}

Maria do Rosário Girão Ribeiro dos Santos

\section{(2) OpenEdition}

\section{Journals}

Édition électronique

URL : http://journals.openedition.org/carnets/1494

DOI : 10.4000/carnets. 1494

ISSN : 1646-7698

Éditeur

APEF

\section{Référence électronique}

Maria do Rosário Girão Ribeiro dos Santos, «L'insularité : du mythe à la réalité », Carnets [En ligne], Deuxième série - 3 | 2015, mis en ligne le 28 février 2015, consulté le 19 avril 2019. URL : http:// journals.openedition.org/carnets/1494; DOI : 10.4000/carnets. 1494

Ce document a été généré automatiquement le 19 avril 2019.

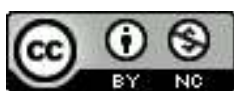

Carnets est mis à disposition selon les termes de la licence Creative Commons - Atribution - Pas d'utilisation commerciale 4.0 International. 


\title{
L'insularité : du mythe à la réalité
}

\author{
Maria do Rosário Girão Ribeiro dos Santos
}

Depuis les temps immémoriaux, l'île s'avère une utopie accueillant le «principe de la conjonction des contraires » et la " relation qui les conjoint dans leur opposition même » (Marin, 1973 : 31) et/ou une 'distopie', un lieu ou un non-lieu, dont on rêve en termes paradisiaques ou dont on s'enfuit à cause de l'hostilité naturelle, un espace euphorique ou disphorique consolidant la soif de l'inconnu qui hante l'être humain. Il suffit de rappeler, à travers le périple du rusé Ulysse, les îles ensorcelées des Lotophages, des Sirènes, de Circé et des Cyclopes, ainsi que cette Atlantide platonicienne engloutie à jamais et jamais retrouvée. Ou, alors, faisant suite à l'odyssée mythique, se souvenir de l'équipée d'Argos en quête de la Toison d'Or, des chevaliers de la Table Ronde en demande du Graal et de la traversée de l'Asie par Marco Polo, qui finit par rejoindre la Chine. À leur tour, le xve et le $\mathrm{xVI}^{\mathrm{e}}$ siècles ne font que renouveler cet imaginaire individuel et collectif, en ouvrant la voie, par les Découvertes, aux îles du Pacifique et à l'Australie et en convainquant les Européens que la culture des nouveaux mondes, loin d'être démonologique, contribue à l'enrichissement anthropologique de l'univers et comble, dans la carte du monde, quelques lacunes géographiques et ethnologiques. Dès lors, la fiction, boulimique, s'approprie l'insula, comme celle, volante, nommée «Laputa ", que Gulliver croise dans son voyage, au long duquel il fait connaissance de géants, d'êtres minuscules et d'une société idéale gouvernée par des chevaux, l'espèce humaine étant considérée méprisable.

Du point de vue génologique, les possibilités d'une île sont multiples, depuis la BD, en passant par le récit de jeunesse et en achevant cet itinéraire d'une simplicité contraignante par le roman d'aventures et le récit de suspense. Qui ne se rappelle pas L'île Noire d'Hergé, d'où le célèbre reporter Tintin et son fidèle chien Milou ramènent le gorille dont la fonction était de terroriser les pêcheurs de la région? Ou, alors, L'île du Sommeil, dont le protagoniste est un jeune garçon de onze ans, Eelian, qui, après un accident de vélo, tombe dans le coma et se retrouve dans une dimension parallèle qui a pour nom « Noctance ", l'île de ses rêves ? Ou, encore, L'île aux perroquets (1942) de Robert Margerit, narrant l'histoire d'Antoine qui embarque sur le Walrus avec un groupe de hors-la-loi, qui, après la découverte d'un trésor (faisant suite à L'île au trésor de Louis Stevenson parue en 1883), deviennent aristocrates pour, à la fin, redevenir pirates? Ou, alors, L'̂́le aux 
peupliers de Daniel Vigoulette, roman empli d'émotions où, dans le paysage inquiétant des bords de la Gironde, Sarah O'Donnell essaye de recommencer sa vie? Ou, pour ne plus citer, L'̂̂le aux sarcasmes, de Pierre Drachline, où la protagoniste, révoltée, décide de divorcer du monde dans une île synonyme de « prison sûre » ?

Dans une perspective thématique, l'île est souvent envisagée en termes de purgatoire et, même, d'enfer... En 1719 (huit ans avant la parution du livre de Jonathan Swift), Daniel Defoe fait paraître La vie et les aventures étranges et surprenantes de Robinson Crusoé, inspiré d'un fait divers, à savoir le compte-rendu d'un voyage autour du monde, publié, en 1712, par le capitaine Woods Rogers, où l'on peut lire sa rencontre, dans l'archipel de Juan Fernandez, au large du Chili, avec l'Écossais Alexandre Selkirk, semblant plus sauvage que les chèvres, qui s'était brouillé avec le commandant du navire « Cinq Ports » et était resté, pendant quatre ans et quatre mois, sur l'île de « Mas a Tierra ». À partir de cet hypotexte, Defoe crée le personnage de Robinson, remplace la mésentente par un naufrage, prolonge la durée du séjour insulaire (vingt-quatre ans en plus), déplace l'aventure du Pacifique à l'Atlantique et invente Vendredi, compagnon d'infortune (ou de la fortune?) du héros solitaire. Les dés sont jetés pour la création du personnage mythique et pour la fondation du mythe littéraire ${ }^{1}$, susceptible d'être défini en tant que configuration narrative symbolique : configuration, étant donné qu'il est constitué par plusieurs mythèmes; narrative, parce qu'il présuppose une mise en intrigue qui doit être dite et redite, reprise et répétée systématiquement, quoiqu'avec des variantes; symbolique, dans la mesure où chaque élément du système, polysémique, dépend de l'ensemble (Chevrel, 2006 : 65-66).

Cela étant, et tout en partant des mythèmes robinsoniens (départ, naufrage, séjour sur l'île et retour), Marivaux, lecteur de Defoe, écrit L'île des Esclaves, comédie en un acte et en prose - à laquelle se succéderont L'île de la Raison (1727) et La Nouvelle Colonie (1729) représentée pour la première fois par les Comédiens Italiens du Roi le lundi 5 mars 1725. Réchappés du naufrage et arrivés à l'île des esclaves, les maîtres Iphicrate et Euphrosine et leurs esclaves Arlequin et Cléanthis sont reçus par Trivelin, qui attire leur attention sur les dangers d'une éventuelle évasion et sur les lois de la République, dont la première est l'inversion des rôles, entraînant, pour celui qui abuse du pouvoir, Iphicrate, le changement d'habit, le dépouillement des symboles du pouvoir (le bâton et l'épée), la perte du nom ou de l'identité et la dépossession de la parole (Jordy, 1991). Dès l'incipit une antinomie significative s'ébauche : d'une part, l'île, tremplin pour la satire, fréquemment désignée par le déictique «ici », apparaît comme un espace resserré et un contrepays orchestré par l'équité; d'autre part, la lointaine Athènes, indigne et censurée, surgit métonymiquement comme un "là-bas", paradigme de liberté pour les maitres et d'indignité pour les esclaves. Quant à la structure de la pièce - exposition, épreuve des portraits et réconciliation -, elle n'aiguise la satire qu'en introduisant la parodie sous la forme de théâtralisation du discours et de la «mise en abîme » qu'est le théâtre dans le théâtre. De fait, les anciens esclaves devenus nouveaux maîtres s'empressent de parodier, en prenant comme modèle leurs anciens maîtres transformés entre-temps en esclaves, le discours social du "grand monde», qui n'est autre que l'aristocratie stéréotypée, parsemé de clichés, de conventions dérisoires, de gestes réglés et codifiés.

5 Cette contrefaçon moqueuse, à valeur psychologique et poétique, met en évidence la vanité et l'insincérité de la "Préciosité », tout en réfléchissant sur l'inanité du langage, traversé de lieux communs, détenant un effet de 'troupeau' et se vidant graduellement de sens. Confrontés avec le miroir de leurs excès comportementaux, gestuels et langagiers, les maîtres reconnaissent leurs torts, les esclaves avouent leurs faiblesses et tous, 
désormais salués, à cause de leur amendement, par Trivelin, se mettent fraternellement ${ }^{2}$ d'accord pour repartir à Athènes ${ }^{3}$, où, dorénavant, les maîtres repentis deviendront tolérants pour leurs esclaves et ceux-ci, honteux, se conduiront en bons serviteurs, car, selon Trivelin, la différence des conditions «n'est qu'une épreuve que les Dieux font sur nous je ne vous en dis pas davantage » (Marivaux, 2004 : 109-110). À ce titre, une tirade d'Arlequin mérite d'être invoquée et convoquée : «Ne dites donc point comme cela, mon cher patron ; si j'avais été votre pareil, je n'aurais peut-être pas mieux valu que vous... » (Marivaux, 2004 : 102). Dans cette conjoncture, serait-ce l'habit qui fait le maître et sa permutation qui le rend esclave? Serait-ce le hasard de la naissance, noble ou plébéienne, le seul responsable de l'attribution de certains ' rôles ' à certains actants sociaux ? Seraitce l'arbitraire du destin l'unique coupable de la hiérarchie sociale? Quoi qu'il en soit, cette île, autorisée à infliger des épreuves punitives par le retournement de la situation, surgit comme « une virtualité d'utopie, une hypothèse dont les conditions de passage à la réalité n'ont pas été remplies » (Coulet, $2004: 23$ ).

Dans ce contexte, nous ne pouvons pas passer sous silence L'île de la Raison et La Colonie, où Marivaux pousse à l'extrême son goût de l'allégorie et "expose en toute nudité ses problèmes de moraliste, ou, pour mieux dire, son problème essentiel : celui de la vérité. » (Arland, 1989: XXXIII). Tout en nous penchant, quoique d'une façon succincte ${ }^{4}$, sur la première, il ne s'avère pas négligeable de constater la dimension moraliste qui s'y étale en profondeur : dans une île non cartographiée, régie par la raison, les hommes deviennent petits ou «myrmidons" quand ils manquent de sagesse et ne reprennent leur taille naturelle que quand ils avouent leurs défauts. Ce duel entre la raison et la folie véhicule soit la métamorphose physique (le rapetissement et le grandissement) soit la métamorphose morale (la guérison des vices), les malades, désormais guéris, devenant médecins, à l'image des insulaires, guérisseurs par excellence. Cela étant, les étrangers se hâtent à assumer leur bêtise et à faire preuve d'humilité : si le paysan Blaise avoue sa fourberie et son ivrognerie et si Gascon Fontignac, secrétaire du courtisan, confesse son 'culte' du mensonge et de la corruption de l'esprit humain, la Comtesse reconnaît, quant à elle, son orgueil et sa coquetterie, tandis que son frère, le courtisan, déclare son hypocrisie. Reprenant la structure de L'île des Esclaves (la comédie dans la comédie), ainsi que la dualité spatiale ( «ici » versus « la patrie ») et la critique des mœurs, L'Île de la Raison ne se révèle inefficace qu'à l'égard du philosophe, qui persévère dans son arrogance, et du Poète, qui insiste sur l'insolence de ses épigrammes. Parallèlement à cette pièce, La Colonie (comédie en un acte et en prose) met en scène, dans une île innommée et désignée par « pays sauvage » (Marivaux, 1989: 646), une émeute féminine qui semble n'avoir d'autre but que celui de la tourner en dérision. En fait, Arthenice, dame de la noblesse, et Mme Sorbin, femme d'artisan, s'unissent contre le noble Timagène et M. Sorbin afin de revendiquer leurs droits, de lutter contre l'avilissement et la soumission qu'elles doivent endurer, tout en réclamant la fin de la servitude et l'égalité entre les sexes. La révolte féminine sera définitivement résolue lors de l'attaque des sauvages qui descendent dans la plaine : «MADAME SORBIN. - (...) Viens, mon mari, je te pardonne ; va te battre, je vais à notre ménage ; TIMAGÈnE. - Je me réjouis de voir l'affaire terminée » (Marivaux, 1989: 671).

7 Si l'île est au service de la satire, notamment de la critique sociale, elle l'est aussi par rapport à la "parodie sérieuse " (se situant, d'après Gérard Genette, à la frontière du ludique et du sérieux), comme c'est le cas de Suzanne et le Pacifique de Jean Giraudoux - qui a été non seulement un critique littéraire ${ }^{5}$ pour lequel le théâtre est, par définition, 
«l'alibi » (Debidour, 1955 : 21), mais aussi le champion de l'hypertexte ${ }^{6}$ - qui, malgré les déplacements, les transpositions et les retournements, reste 'fidèle' aux mythèmes consacrés par la tradition. Cela étant, on assiste, de prime abord, à la transexuation ou féminisation de Robinson, qui naît en 1632 (Defoe, s/d: 9) et qui débarque sur lîle du Désespoir en 1659 (Defoe, s/d : 77) : Suzanne, l'héroïne éponyme du roman, est une jeune fille de Bellac ${ }^{7}$, âgée de dix-huit ans, sans nom ni métier ni famille, qui gagne un voyage autour du monde ${ }^{8}$ offert par le Sydney Daily lors de son « concours de la meilleure maxime sur l'ennui » (Giraudoux, 1990 : 478). Ensuite, la translation spatiale n'est pas du tout négligeable : de l'Atlantique on passe au Pacifique, plus concrètement à une île utopique ${ }^{9}$ et dérisoire - «mon île » ${ }^{10}$ - qu'un marin baptise « île Suzanne » et qui semble illustrer le mythe rousseauiste $\mathrm{du}$ « bon sauvage ». À ses côtés se dressent deux îlots, dont l'un - l'île des dieux - serait, selon le témoignage giralducien, l'île de Pâques (Gauvin, $1990: 1559$ ). D'ailleurs, l'onomastique de l'archipel est repassée d'un fin sens de l'humour ${ }^{11}$, comme on le constate dans une lettre écrite par Suzanne à Simon : «Sur le carnet du naufragé d'en face, j'ai trouvé le plan de mon archipel (...) le nombre exact de milles qui le séparent de l'île Palmyre (...) de l'île Rimski-Korsakov (...) et de la Rakahanga (...)» (Gauvin, 1990: 585). Une différence cruciale, au niveau de la géographie et de la topographie insulaires, s'impose d'ores et déjà: tandis que l'île de Robinson, à l'embouchure de l'Orénoque, n'est guère transcendantale, devenant peu à peu l'espace de la conquête humaine, les îles de Giraudoux, où règne un temps éternel s'apparentant au temps mythique, sont tiraillées entre l'univers naturel des oiseaux (Gauvin, 1990: 514) et l'immortalité des dieux, " alignés par centaines comme des menhirs » (Gauvin, 1990 : 542). Dans cette conjoncture, la transposition thématique s'avère d'une importance extrême. En effet, si la relation, chez Defoe, entre la nature et la culture est vécue sur le mode de l'opposition, elle apparait, dans le roman de Giraudoux ${ }^{12}$, sous l'égide de l'harmonie totale. L'artisan Robinson, imbibé du désir de civilisation, de l'apologie de la raison, de l'éloge du travail et de l'idéologie utilitariste qui caractérisent le xvIII siècle, se transforme, au fil du temps, en agriculteur, en moissonneur, en menuisier, en potier, en meunier et en éleveur de chèvres. Ainsi, apprivoise-t-il l'île à son échelle, en accumulant des pièces artisanales, en entassant des récoltes, enfin, en transposant la civilisation anglaise en un lieu sauvage, un non-lieu qu'il façonne de bon gré comme son lieu. Différemment du héros de Defoe ${ }^{13}$, Suzanne conteste le travail, réfute la raison, étale sa nudité de " fille-oiseau », protège son île d'un alligator et d'un cougouar et constate que la faune et la flore de son habitat insulaire lui font cadeau, depuis le naufrage, d'un changement contre nature. Cela étant, les pommiers donnent des oranges, les figuiers des cerises et les poissons couvent... (Gauvin, 1990 : 579).

8 En outre, pendant le temps où elle subit les "tortures de l'attente » (idem: 510) et s'adonne avec luxure au rêve, elle crée et recrée l'univers et s'amuse à se dédoubler: "L'une [Suzanne] est capable de tous les exploits, l'autre [Suzanne] de toutes les bassesses. L'une est idolâtre, crédule; l'autre raisonne. (...) L'une innocente, l'autre perverse (...)» (Gauvin, 1990 : 589). Ainsi n'a-t-elle pas besoin, à l'image de Robinson, de Vendredi, avec lequel elle s'identifie en ce qui concerne la fusion avec l'univers ou le passage de l'antithèse nature/culture à l'unité primordiale. Nouvelle Ève dans le paradis polynésien (Gauvin, 2000 : 91-99), elle s'apparente, génératrice de mythes, à une sirène, à une walkyrie, à Nausicaa et à Yseult - n'est-elle pas amoureuse de Tristan/Simon ? - pour devenir, enfin, Alice au pays des merveilles (Gauvin, 2000 : 535). Quant à Robinson, on peut deviner sa présence en filigrane derrière ce naufragé qui avait précédé Suzanne dans l'île et dont les traces centenaires et périssables sont signalées soit par la répétition 
constante du déictique «ici» (renvoyant à une nature prodigue), soit par l'insistance frénétique sur le pronom «il», se rapportant à la vacuité du travail et du caractère gratuit de l'effort herculéen.

(...) ici, où tout est abondance en fruits et en coquillages, il avait défriché et semé du seigle ; ici, près de deux grottes chaudes la nuit et fraîches le jour, il avait coupé des madriers et bâti une hutte; ici, où l'on apprend à grimper en deux heures, il avait construit des échelles (...) ici, où les ruisseaux coulaient à vitesse différente pour étancher les soifs les plus diverses, il avait amené des conduites en bambou jusqu'à sa case ; ici, où partout était la mer, il y avait une petite piscine en ciment (...) (Gauvin, 2000 : 539).

9 À vrai dire, Suzanne est antirobinsonienne surtout dès le moment où, à travers une mise en abîme magistrale portant sur le parallélisme spéculaire entre son sort funeste et celui de son précurseur mythique, elle commence à lire le roman de Defoe (où le lexème «Providence $~^{14}$ est récurrent) et étale, une fois la lecture achevée, son indignation totale en ce qui concerne sa conduite, tout en s'empressant d'affirmer : « Le seul homme, peutêtre, tant je le trouvais tatillon et superstitieux, que je n'aurais pas aimé rencontrer dans une ̂̂le. » (Gauvin, 2000 : 582-583).

De même, dans une belle séquence anaphorique introduite par quatre paragraphes commençant par «Ce n'est pas vrai » ${ }^{15}$, Suzanne s'entête à mettre en cause et à démentir les vérités affichées ou, en d'autres termes, à démythifier le mythe, tout en se positionnant aux antipodes des autres naufragés : «Je rougis d'avouer à quoi se passa ma première semaine, quand je compare cette vie frivole à celle des naufragés classiques. » (Gauvin, 2000 : 509). Le temps passe (cinq, six ans), la guerre éclate, le Lusitania coule et Suzanne est sauvée, sans exposer aux yeux d'autrui sa « demeure d'objets qui pouvaient être utiles, mais qui l'eussent ridiculisée le jour de [son] sauvetage, tables, chaises ou baquets " (Gauvin, 2000: 603). Ce qu'elle cherche, au fond, et ce qui définit soit son voyage atopique et initiatique (quoique circulaire, comme celui de Robinson) soit son naufrage devenu renaissance, c'est la quête de soi-même, de son nom et, aussi, du mot. Au début du chapitre IV, qui correspond au débarquement et à la conscience de son insularité, elle se nomme : “'Suzanne!' criai-je. » (Gauvin, 2000 : 506). Au chapitre IX, dans une lettre adressée à Simon, elle raconte qu'elle a inventé une langue à elle seule, dépouillée de ces $h$ aspirés qu'elle déteste : «Langage sans suffixes, ni préfixes, ni racines, où les êtres qui se ressemblent le plus ont les noms les plus différents. Noms sifflants toujours suivis d'une belle épithète qui les nourrit comme un tender." (Gauvin, 2000 : 588). Finalement, les plusieurs noms des auteurs et les nombreuses références littéraires ${ }^{16}$ qui traversent le roman rendent le voyage de Suzanne littéraire, construisent, à partir de l'île, une métaphore du langage, moins du saussurien signe arbitraire que d'une conscience cratylienne traduite par la rêverie nominale, et classent l'œuvre giralducienne, dont l'écriture est visuelle et picturale, comme un antiroman, un «nouveau roman » et une aventure du récit. Dans cet univers littéraire, il est curieux de rappeler que le marivaudage ne passe pas sous silence: «Derrière toutes ces manies et ces cadres de l'esprit et de l'âme, dont nous répétions les noms au pensionnat comme des perroquets : Scolastique, Marivaudage, Préciosité... à l'aide de vieux syllogismes (...)» (Gauvin, 2000 : 556).

11 Or, quarante-six ans après Suzanne et le Pacifique (roman publié en 1920-1921), Michel Tournier (qui n'est pas « très méditerranéen $»^{17}$ ) publie Vendredi ou les limbes du Pacifique ${ }^{18}$, réécriture de Robinson Crusoé comportant des échos leibniziens (la monade qui exprime le monde) et sartriens (la théorie d'autrui, selon laquelle celui-ci, objet et sujet, devient une 
structure qui précède le regard) : en vérité, dans la nuit du 29 au 30 septembre 1759, le capitaine Van Deyssel, qui commande le bateau Virginie, tire les cartes à un passager dénommé Robinson et lui prédit (une prédiction qui ne tardera pas à se réaliser...) sa vie future sur l'île. Le bateau ayant choqué contre un obstacle, Robinson parvient à se sauver, conjointement avec le chien de bord Tenn, se réveille sur la plage d'une île - qu'il désigne, d'abord, par « île de la Désolation » et, ensuite, par «Speranza » (Tournier, 1981: 45) -, organise son temps, s'approprie l'espace, édicte une "Chartre » et un "Code pénal», rencontre Vendredi et, après un séjour de vingt-huit ans, deux mois et dix-neuf jours, renonce à partir avec les marins d'une goélette anglaise (Tournier, 1981 : 243), découvre la trahison de son compagnon et vérifie que le petit mousse du Whitebird a choisi de rester avec lui : « Désormais, lui dit Robinson, tu t'appelleras Jeudi. C'est le jour de Jupiter, dieu du Ciel. C'est aussi le dimanche des enfants. » (Tournier, 1981: 254). Soulignons, à ce propos, la transvalorisation et la revitalisation ${ }^{19}$ du mythe moyennant la non-circularité du roman (Robinson reste sur l'île) et l'intrusion de l'avatar de Vendredi, Jeudi, dont le prénom cesse de se reporter à Vénus. Dans cet ordre d'idées, la transvocalisation - le passage du récit de la première à la troisième personne - paraît annoncer la principale transformation thématique consistant en un retournement idéologique corroboré par le pacte de lecture établi par le titre ${ }^{20}$. En fait, le héros de Defoe, qui avait désobéi à son père en choisissant la carrière de marin, envisage le microcosme qu'est l'île presque comme un purgatoire (dans l'explicit il la rend riche et devient un homme réussi), procède à l'exploitation de ses ressources ainsi qu'à l'inventaire des richesses graduellement acquises (à l'image du macrocosme Angleterre au seuil de la révolution industrielle), prend refuge dans le travail comme un moyen de rédemption et étale une attitude rétrospective, visible dans la nostalgie d'un temps, antérieur à l'industrialisation croissante, où l'être humain était capable de maîtriser diverses techniques au lieu de se spécialiser dans un seul domaine. Par contre, le Robinson de Tournier, loin d'être un héros positif (selon Georg Lukács), s'avère un héros problématique, s'efforce d'emprisonner le Temps par la voie d'une clepsydre et thésaurise pour fuir ses moments de défaillance, durant lesquels la folie le menace et le langage, entre-temps 'déconstruit', lui fait défaut (Tournier, 1981: 68). Son séjour, d'ailleurs, est le tremplin pour une métamorphose ${ }^{21}$ croissante : faisant suite aux doutes qui l'assaillent et aux hallucinations qui s'emparent de lui, il se fond et se confond avec "Speranza», il s'ensevelit dans l'alvéole comme un fotus dans le ventre maternel (regressus ad uterum) et il renonce à y redescendre, tout à fait conscient de la relation incestueuse de l'homme qu'il est devenu avec l'île mère qui le nourrit : « Alors Robinson est Speranza. (...) Par quelle aberration aije pu me prévaloir de l'innocence d'un petit enfant? Je suis un homme dans la force de l'âge et je me dois d'assumer virilement mon destin.» (Tournier, 1981: 98-114). La tentation maximale est la " souille », « la mare boueuse » (Tournier, 1981 : 49) ou la fange liquide, un véritable enfer à nuances paradisiaques, à la fois séductrice et dangereuse, dans les maelstroms de laquelle il faillit tomber et retomber : "Chaque homme a sa pente funeste. La mienne descend vers la souille. (...) La souille est ma défaite, mon vice. » (Tournier, $1981: 50$ ). En outre, son accord total avec la nature va plus loin que celui de Suzanne dans le roman homonyme. L'île apparaissant comme une épouse, il tente une relation sexuelle, dans un premier moment, avec un arbre et, ensuite, avec une combe, les mandragores, à son avis, devenant les fruits de cette union, voire ses enfants (Tournier, 1981: 137). Néanmoins, l'originalité de ce roman initiatique porte sur les relations inédites entre le «Gouverneur » Robinson et son sauvage, qu'il traite en fils adoptif : de prime abord, celui-ci considère le premier comme son seigneur, feignant une soumission 
extérieure, puisque fallacieuse ; ensuite, le déroulement de l'intrigue nous apprend que Vendredi, surpris par son soi-disant maître à fumer le tabac de ce dernier, jette la pipe sur les tonneaux remplis de poudre noire qui explosent et détruisent toute la production de Robinson; la suite des événements inaugure les nouveaux rapports entre l'homme occidental et l'indien : d'une part, un « nouveau Robinson se débattait dans sa vieille peau et acceptait à l'avance de laisser crouler l'île (...)» (Tournier, 1981: 189; d'autre part, « Vendredi allait l'entraîner vers autre chose. » (Tournier, 1981 : 189). Parallèlement à L'Île des esclaves, les rôles sont renversés, ce renversement débouchant sur une duplication d'identités, la vraie et la fausse, qui va de pair avec une île double que le déterminant indéfini rend symptomatique - une « autre île » (Tournier, $1981: 181)$.

Dès lors ils vécurent à quatre sur l'île. Il y avait le vrai Robinson et la poupée de bambou, le vrai Vendredi et la statue de sable. (...) Si Vendredi était Robinson, le Robinson de jadis, maître de l'esclave Vendredi, il ne restait à Robinson qu'à devenir Vendredi, le Vendredi esclave d'autrefois. (...) Ils jouèrent souvent à ce jeu. (Tournier, 1981 : 211-212-213).

D'initiateur tellurien Robinson passe à initié éolien à travers Vendredi - « une nature » aérienne -, qui s'apparente aux héros mythiques. En effet, il vit le carpe diem (Tournier, 1981 : 190), pendant lequel il s'adonne, insouciant, au rire, au jeu et à la danse (Tournier, 1981 : 153-192-221-222). Imbibé de sentiments contradictoires - il aime passionnément le chien et il torture, d'une façon implacable, une tortue -, Vendredi apprend à Robinson que la vie du corps est primordiale, lui enseignant, également, à adresser ses prières au Soleil, remplaçant, de la sorte, Dieu et la Bible par l'astre diurne et faisant preuve d'un panthéisme indéniable : « Soleil, délivre-moi de la gravité. Lave mon sang de ses humeurs épaisses (...) Soleil, es-tu content de moi ? Ma métamorphose va-t-elle assez dans le sens de ta flamme? » (Tournier, 1981 : 191-217).

Une fois la métamorphose accomplie, Robinson, ayant une attitude prospective, devient un Robinson de soleil dans une île devenue elle-même apollinienne, un être uranien dans Uranus, et se distingue, d'après Gilles Deleuze (Deleuze: 1981: 259-260), du héros mythique par trois traits flagrants : il ne se rapporte pas à une origine, mais plutôt à des fins; ces buts ne traduisent pas la reproduction économique de notre monde, dont l'emblème est le travail, mais une déviation fantastique, à l'ombre d'une sexualité transformée, pas du tout conforme à l'univers. Un doute, pourtant, se dresse à l'horizon : Robinson serait-il l'incarnation de la perversité, de la pureté ou de l'innocence ? Si la pureté est le renoncement au monde et si l'innocence consiste dans son heureuse acceptation, la perversité, encore selon Deleuze, renvoie à l'introduction du désir « dans un tout autre système et lui fait jouer, dans ce système, le rôle d'une limite intérieure, d'un foyer virtuel ou d'un point zéro » (Deleuze, 1981 : 261).

Si le mythe littéraire se prête soit au roman d'avatars, comme c'est le cas de Vendredi ou les limbes du Pacifique, dont on peut faire une lecture psychanalytique - selon Deleuze, Robinson passe de la névrose (symbolisée par la "souille») à la psychose (traduite par l'ensevelissement dans la grotte) et à la perversion (illustrée par sa vénération du soleil -, philosophique (la place d'Autrui dans la conscience et dans l'existence de l'être humain) et ethnologique (se basant, dans la lignée de Claude Lévi-Strauss, sur la rencontre et la fusion de deux cultures), soit au roman poétique Suzanne et le Pacifique, ayant comme but, moyen et fin le langage, soit au roman d'aventures, emblématisé par les péripéties de L'Île aux perroquets, soit à la comédie détenant des scènes de farce populaire, des «zannis » et d'autres personnages issus de la tradition de la "commedia dell'arte», il s'approprie, également, la poésie à valeur allégorique. Sur ce sujet, il serait impensable de ne pas faire 
une référence au poème «Fábula » de Francisco Bugalho - publié dans la revue presença [en 1937] -, dans lequel on constate l'absence de Vendredi colmatée par la présence symbolique des perroquets, qui accomplissent leur mission de partir et de laisser, par conséquent, Robinson dans la plus complète solitude.

Il faut, cependant, souligner que le naufrage devient, parfois, intérieur... En effet, les 'naufragés de la vie' peuvent ne pas lire la Bible (comme Crusoé et le Robinson de Vendredi ou les limbes du Pacifique), ne pas tenir un journal intime (comme le héros de Defoe) ou un log-book (à l'image du héros de Tournier), ne pas écrire des missives (comme Suzanne à Simon), mais sacralisent leur existence à travers l'écriture, qui reflète leurs plus intimes obsessions existentielles: «Escribir significa transformar la vida en pasado, o sea envejcer " (Vila-Matas, 2006: 228). En l'occurrence, le voyage et l'île deviennent, dans l'œuvre de Vila-Matas ${ }^{22}$, initiaques, sous l'égide de l'autofiction, du recours constant aux doubles, du métadiscours incessant, de l'écriture comme forme de vie, de l'étrangeté relative au quotidien et de la fuite au réalisme par la voie de l'intrusion de quelques scènes inspirées de Kafka. Arrêtons-nous, en conséquence, sur le roman de formation $\mathrm{El}$ Viaje Vertical: le protagoniste septuagénaire, Frederico Mayol (le/un double de VilaMatas ?), chassé de chez lui par son épouse Julia, père de Julian qui est convaincu d'avoir été, dans une vie antérieure, un habitant de l'Atlantide, obsédé par le caractère contradictoire de l'homme et par l'imminence de la mort, traumatisé par l'interruption définitive de ses études due à la guerre civile espagnole, affligé par un cauchemar récurrent, à savoir son séjour dans un hôtel où il ne paie jamais, dicte l'histoire de son exil sans retour ou de sa nouvelle vie au narrateur - présence qui s'insinue au début et se convertit en personnage principal -, décide de visiter des villes inconnues et de se déplacer jusqu'à l'̂̂le de Madère, tremplin pour sa métamorphose, où il assiste à quelques communications d'un Colloque international. Dans un premier temps, Mayol, incultivé, prend des notes, un peu au hasard, faisant preuve d'un humour indéniable qui côtoie, souvent, l'ironie...

16 Néanmoins, une fois son séjour insulaire achevé et son identité menacée rebâtie, l'autodidacte Mayol devient cultivé et artiste et se consacre à la «culture sans discipline », au point de croire, comme Julian, à l'Atlantide ensevelie. C'est alors qu'il est invité à donner des conférences sur les îles et la mythologie, invitant son lecteur à passer de l'autofiction à la réalité. En vérité, Mayol/Enrique Vila-Matas a donné la conférence de fermeture, sur "Espionnage et Littérature», du Colloque international "As Ilhas e a Mitologia ", qui a eu lieu à Funchal, au théâtre Baltazar Dias, dans l'année lointaine de 1997... D'ailleurs, nous étions là.

17 En guise de conclusion, on peut dire que les possibilités d'une île sont innombrables, soit du point de vue génologique (roman, théâtre, poésie, bande dessinée), soit dans une perspective heuristique (espace paradisiaque/utopique et/ou « huis-clos » distopique) et herméneutique (psychanalytique, sociologique et thématique). Espace fermé, non exempt de périls, dénotant l'isolement et la fermeture (quoique la mer s'apparente à l'infini), l'île, d'après les réécritures analysées de Robinson Crusoé, devient le lieu par excellence de correction (car elle est punitive et vengeresse), d'apprentissage et de formation (puisque l'être humain apprend à y survivre), ainsi que de sagesse et d'initiation (car elle met en œuvre la prise de conscience des 'naufragés de la vie'). En outre, elle est tremplin non seulement pour la satire sociale (comme c'est le cas de la 'trilogie insulaire' de Marivaux) et pour la "parodie sérieuse » (les aventures de la giralducienne Suzanne), mais aussi pour la rédemption et la découverte de soi, après la descente aux enfers (Robinson et 
Mayol). Enfin, un lieu de connaissance pour les néophytes ou les initiés étrangers, entretemps devenus insulaires...

\section{BIBLIOGRAPHIE}

ARLAND, Marcel (1989). « Préface ». Théâtre Complet de Marivaux. Paris : Gallimard, pp. IX-LV.

BODY, Jacques (1986). Jean Giraudoux. La légende et le secret. Paris : puf écrivains.

BODY, Jacques (1990). «Introduction générale ». Euvres romanesques complètes de Jean Giraudoux.

Paris : Gallimard, pp. IX-XXXIX.

BUGALHo, Francisco (1993). « Fábula », presença, vol. III, nº49, p. 10.

CHEVREL, Yves (2006). La littérature comparée. Paris : puf.

Colin, Fabrice (2011). L'T̂le du sommeil. Paris : Flammarion.

coulet, Henri (2004). « Préface » de L'T̂le des Esclaves. Paris: Gallimard, pp. 7-36.

DEBIDOUR, Victor-Henry (1995). Jean Giraudoux. Paris : Éditions Universitaires.

DEFOE, Daniel (s/d.). A vida e as aventuras de Robinson Crusoé. Lisboa : Portugália Editora, traduction de Pinheiro Chagas.

DELEUZE, Gilles (1981). « Michel Tournier et le monde sans autrui ». « Postface » de Vendredi ou les limbes du Pacifique. Paris : Gallimard, pp. 257-283.

DELEUZE, Gilles (2004). A Ilha deserta e outros textos. Textos e entrevistas (1953-1974). São Paulo :

Editora Iluminuras.

DRACHLINE, Pierre (2007). L'T̂le aux sarcasmes. Paris : Flammarion.

GAUVIN, Lise (1990). Notice sur Suzanne et le Pacifique. Paris : Gallimard, coll. « Bibliothèque de la Pléiade », pp. 1549-1569.

GAUVIN, Lise (2000). « Suzanne et ses sœurs : dérivation d'un mythe ou Jean Giraudoux entre le mythe et l'utopie » in Sylviane Coyault, Pierre Brunel, Alain Duneau, Michel Lioure (orgs.).

Giraudoux et les mythes. Paris : Presses Universitaires Blaise Pascal, pp. 91-99.

GAZAGne, Paul (1954). Marivaux par lui-même. Paris : Seuil.

GENETTE, Gérard (1992). Palimpsestes. La littérature au second degré. Paris : Seuil.

GIRAUDOUX, Jean (1980). Les cinq tentations de La Fontaine. Paris : Bernard Grasset.

GIRAUDoux, Jean (1990). Suzanne et le Pacifique. Euvres romanesques complètes. Paris : Gallimard, coll. «Bibliothèque de la Pléiade ».

GIRAUDOUX, Jean (1994). Littérature. Paris : Gallimard.

GOULDING, Elizabeth (2000). « Giraudoux, mystificateur du Pacifique » in Sylviane Coyault, Pierre Brunel, Alain Duneau, Michel Lioure (orgs.). Giraudoux et les mythes. Paris : Presses Universitaires Blaise Pascal, pp. 161-171. 
HUET-BRICHARD, Marie Catherine (2001). Littérature et Mythe. Paris : Hachette Supérieur.

JORDY, Jean (1991). L'T̂le des Esclaves. Paris : Bertrand-Lacoste.

KERAUTRET, Michel (1997). La littérature française du XVIII . Paris : Presses Universitaires de France.

MARGERIT, Robert (1997). A Ilha dos Papagaios. Lisboa : Editorial Bizâncio, coll. « Ilhas Encantadas », traduction d'Ana Maria Machado Chaves.

MARIN, Louis (1973). Utopiques jeux d'espaces. Paris : Éd. de Minuit.

MARIVAuX, Pierre Carlet de (1989). CEuvres. Théâtre. L'T̂le de la Raison ou Les Petits Hommes. La Colonie. Paris : Gallimard, coll. « Bibliothèque de la Pléiade », pp. 483-548, 637-671.

MARIVAUX, Pierre Carlet de (2004). L'T̂le des Esclaves. Paris : Gallimard, édition d'Henri Coulet. SBIROLI, Lynn Salkin (1987). Michel Tournier : La séduction du jeu. Genève-Paris : Éditions Slatkine. STIRN, François (1992). Vendredi ou les limbes du Pacifique. Paris : Hatier.

TOURNIER, Michel (1981a). Vendredi ou les limbes du Pacifique. Paris : Gallimard.

TOURNIER, Michel (1981b). « Qu'est-ce que la littérature ? Un entretien avec Michel Tournier », magazine littéraire. Julien Gracq, ํㅡ179, pp. 80-86.

TOURNIER, Michel (1987). Vendredi ou la vie sauvage. Paris : Gallimard.

VIGouletTe, Daniel (2006). L'île aux peupliers. Paris : Albin Michel.

VILA-MATAS, Enrique (2006). El viaje vertical. Barcelona : Editorial Anagrama, coll. « Quinteto ».

VRAY, Jean-Bernard (1997). Michel Tournier et l'écriture seconde. Lyon : Presses Universitaires de Lyon.

\section{NOTES}

1. $c f$. , en ce qui concerne les rapports entre le mythe et la littérature, la définition de HuetBrichard: «Mythe et littérature ont la même relation médiatisée avec le réel: ils l'intègrent grâce aux processus de symbolisation dans un discours qui a ses lois, son organisation, et qui leur donne sens. Il n'y a donc pas de mensonge ou de vérité du mythe ou de la littérature : tous deux proposent du réel une lecture qui a sa cohérence et sa logique propres. » (2001:37-38).

2. À ce propos, et selon Paul Gazagne, «L'organisation idéale de la société humaine ne dépend pas des contributions ou des lois qui la régissent, mais elle est surtout tributaire d'une vertu que Marivaux place au-dessus de toutes les autres : la fraternité » (1954:144).

3. D'après Michel Kerautret, «Marivaux n'ignore pas la société de son temps (...) et ses personnages ne se 'mésallient' presque jamais, la réalité des classes sociales s'imposant à eux jusque dans les îles des pièces utopiques, où tout finit par retrouver son ordre antérieur » (1997 : 19).

4. Nous avons privilégié, en ce qui concerne notre analyse, L'T̂le des Esclaves, étant donné que cette pièce est bel et bien une réécriture de Robinson Crusoé, tandis que L'Île de la Raison semble avoir comme hypotexte Gulliver's Travels, roman satirique écrit par Jonathan Swift en 1721: «Le Marquis, avec précipitation. - Quoi! sérieusement tu crois qu'il n'y est pas question [dans la comédie à jouer] de Gulliver? (...) je serais fâché cette heure que dans la comédie que nous allons voir on eût pris l'idée de Gulliver ; (...)» (1989 : 488 et 490). En outre, si La Colonie échoua et ne fut pas imprimée, si Marivaux avoua, dans la «Préface » de L'T̂le de la Raison, qu'il avait eu tort à 
donner "cette comédie-ci au théâtre " (1989: 485), L'île des Esclaves connut un succès considérable, eut vingt et une représentations consécutives et fut souvent reprise.

5. $c f$., à ce propos, ses conférences sur Les cinq tentations de La Fontaine, la première étant la tentation de la vie bourgeoise, la deuxième celle du mundus mulierum, la troisième celle de la vie mondaine, la quatrième celle de la littérature et, finalement, la cinquième celle « des deux formes les plus aigues de l'intelligence et de la passion, le scepticisme et la doctrine » (1980:16).

6. D’après Jacques Body (1986: 70-71), Giraudoux a convoqué les tragiques grecs pour Electre, la Bible pour Judith et Sodome et Gomorrhe, l'Odyssée pour Elpénor et l'Iliade pour La guerre de Troie n'aura pas lieu.

7. Notons au passage que Giraudoux, dans son texte intitulé «Bellac et la tragédie », définit celleci comme étant « l'affirmation d'un lien horrible entre l'humanité et un destin plus grand que le destin humain » (1994:249).

8. L'une des escales de ce voyage est l'île de Madère, dont la description nous semble fort intéressante: "Le lendemain parut Madère (...) Déjà ce n'était plus l'Europe. (...) les arbres étaient couleur de saule, le gazon bleu, les ruisseaux rouges. Les mendiants assiégeaient les églises, les vieillards comptant sur ceux qui entrent, les enfants ignorants sur ceux qui sortent » (1990 : 498-499).

9. Attiré par la mythologie, Giraudoux démythifie dans Elpénor les exploits d'Ulysse et, à partir de l'Utopia, créée par Thomas More, il invente, dans Simon le Pathétique, le mot « Outopia » (1990: 289).

10. Il est intéressant de constater que l'île, pour Giraudoux, c'est, comme le souligne Elizabeth Goulding, « un mot de passe, un talisman (...) le lieu de l'enchantement » (2000: 162).

11. Le sens de l'humour giralducien traverse, à vrai dire, tout le roman, depuis les noms des îles (Palmyre est une oasis du désert syrien et Rimski-Korsakov est un compositeur) jusqu'au nom de l'animal que Suzanne découvre dans l'île : « un bel ornithorynque » $(1990: 518)$.

12. Notre choix a porté sur ce roman, non seulement parce qu' il est une réécriture indéniable du mythe de Robinson, mais aussi parce qu'il ouvre l'ère des héroïnes dans la carrière de ce romancier de jeunes filles qui écrit pour les femmes - Juliette au pays des hommes (1924), Bella (1926), La Grande Bourgeoise (1927) et sa sœur de lait Églantine (1927) et, pour ne plus citer, Stéphy (1927). Voir, sur ce sujet, Body, 1990 : XX.

13. Selon Deleuze (2004 : 9-10), tandis que Suzanne et le Pacifique renforce la séparation des îles (et il y a des îles continentales et des îles dérivées), Robinson Crusoé rehausse la création. En effet, si l'île de Defoe débouche sur la reconstitution de la vie quotidienne bourgeoise, la giralducienne île déserte fournit à la protagoniste tout ce que la civilisation a produit pendant des siècles, en lui donnant le double, inconsistant et séparé du réel, de tous les objets de la ville...

14. Giraudoux semble mettre au ridicule la Providence de Defoe/Crusoé moyennant une alliance de mots qui semble contradictoire: «Pour que tout malentendu fût dissipé aussi entre la Providence des parfums et moi, la brise me vaporisait de toutes les odeurs de l'île » $(1990: 508)$.

15. «Ce n'est pas vrai qu'un navire passa, un matin, à peu de milles ; (...) Ce n'est pas vrai qu'alors je voulus mourir de faim. (...) Ce n'est pas vrai que j'usais mes jours à me poncer les jambes et à les frotter d'une poudre de nacre qui les rendait d'argent même sous les rayons du soleil. (...) Ce n'est pas vrai que j'embrassais l'ornithorynque ; (...) » (idem : 518-519).

16. On peut citer, entre autres, Racine, Fénelon, Baudelaire, Shakespeare (1990: 512), Jules Verne (idem : 514), La Fontaine (idem : 537), Corneille, Ronsard, Malherbe, Lamartine, Vigny, Mallarmé, Claudel et Rimbaud (idem : 557).

17. Il s'agit des propos recueillis par Jean-Jacques Brochier dans un entretien avec Michel Tournier. Il vaut la peine d'en citer un extrait: "Pour moi, la mer c'est l'océan, et particulièrement la marée basse. (...) La mer, c'est la marée basse, les grandes étendues de sable mouillé, avec des varechs, des rochers immergés, des reflets, des vases » (Tournier, 1981 : 83-84). 
18. D’après ce roman publié en 1967, Tournier écrit par la suite une version pour les enfants intitulée Vendredi ou la vie sauvage.

19. Pour Jean-Bernard Vray (1997 : 292), l'explosion est le point de l'hypertexte à partir duquel s'accomplit la rupture avec l'hypotexte de Defoe. Si la première ligne de dérive, par rapport à Robinson Crusoé, est le meurtre du bouc introduisant le thème tourniérien de la violence et de la mort, le deuxième décalage est l'oisiveté du protagoniste de Tournier, fasciné par la «souille ", épisode qui infléchit le sens du récit.

20. Il semble, en fait, que Tournier part du modèle mythique pour le gauchir, en prêtant à l'aventure de son naufragé une dimension intérieure qui n'est autre que la menace de la folie.

21. Du point de vue de Lynn Salkin Sbiroli (1987 : 61-62), la métamorphose de Robinson II suit les étapes fondamentales de toute initiation vouée à la révélation des 'secrets' du monde sacré, la première étant l'isolement de l'initié, la deuxième se déroulant selon deux mouvements principaux, la descente aux enfers, la montée au ciel, ou les deux à la fois, et la troisième, l'initiation du 'chaman' ou du 'medecine man', s'avérant une étape réservée à peu de membres de la tribu.

22. Enrique Vila-Matas, inlassable voyageur et touriste invétéré, n'hésite pas à rendre hommage à l'archipel des Açores et à l'île de Madère. En fait, dans Desde la ciudad nerviosa (2000) il se rappelle son séjour inoubliable à S. Miguel et à Faial, en revisitant dans El mal de Montano (2002) livre écrit par un homme atteint de la maladie de la littérature pour ceux qui sont littérairement malades - les anciens baleiniers et le volcan du Pico.

\section{RÉSUMÉS}

Depuis la nuit des Temps, l'île s'avère un espace protecteur et dangereux à la fois, un endroit par excellence de péripéties fabuleuses, un « huis clos » ou un tremplin pour l'imaginaire exotique. Il suffit de rappeler l'odyssée du rusé Ulysse ou, alors, les aventures de Gulliver chez les Liliputiens, la condamnation à vingt-huit ans de solitude de Crusoé et le séjour de Suzanne dans son île du Pacifique. Parallèlement à L'île des Esclaves de Marivaux et à la giralducienne Suzanne et le Pacifique , ces deux œuvres débutant par un naufrage, Enrique Vila--Matas, naufragé de la vie, n'hésite pas à rendre hommage à l'archipel des Açores et à l'île de Madère. Par le biais de son double Mayol, il part à la découverte de soi-même, essayant de rebâtir son identité menacée.

Since the beginning of Time, the island has revealed itself as a protective and dangerous space, at the same time the scene of fabulous adventures, an "huis clos" or a springboard to the exotic imagination. Let's just think of the odyssey of the cunning Ulysses or Gulliver's adventures among the Lilliputitians, Crusoe's condemnation to twenty-eight years of solitude and Suzanne's experience in a Pacific island. Together with L'île des Esclaves by Marivaux and the Giraudoucian Suzanne et le Pacifique, two novels that start with a shipwreck, Enrique Vila-Matas, a real life castaway, does not hesitate in paying hommage to the archipelago of The Azores and the Madeira Island. He sets off, through his double Mayol, at the discovery of his own self, trying to rebuild his threatened identity. 
INDEX

Keywords : island, shipwreck, imagination, satire, parody Mots-clés : île, naufrage, imaginaire, satire, parodie

\section{AUTEUR}

MARIA DO ROSÁRIO GIRÃO RIBEIRO DOS SANTOS

Université do Minho

rosario[at]ilch.uminho.pt 